\title{
EFFECT OF CORTISONE UPON THE VASCULARIZATION WHICH FOLLOWS CORNEAL BURNS
}

\section{HEAT BURNS}

BY

\author{
A. LISTER AND D. P. GREAVES \\ From the Institute of Ophthalmology, London
}

THE purpose of this investigation was to ascertain, primarily from the clinical point of view, whether the vascularization and scarring which follow ocular burns can be inhibited by cortisone, and, if so, whether the use of this drug for this purpose is justified in clinical practice. The experiments reported apply to burns caused by heat; it is proposed to carry out a further series on burns caused by chemicals. Similar investigations were carried out by Jones and Meyer (1950), who found that cortisone caused an inhibition of vascularization after chemical injury to the cornea.

\section{Method}

It was decided that the most satisfactory means of producing a standard lesion was the diathermy current. In order to produce a lesion resembling as nearly as possible an accidental burn, a trial was first made of sparking the dried cornea. This, however, was found to produce variable results compared with those obtained by contact burns using the bipolar current. The animal (rabbit) was anaesthetized with intravenous nembutal and pantocaine drops; the shaved inner surface of one ear was applied to the passive electrode by a standard weight; to the cornea, which had been previously moistened with distilled water, a $1.5-\mathrm{mm}$. (Weve) surface electrode was applied with moderate pressure, $2 \mathrm{~mm}$. from the upper limbal edge, for $12 \mathrm{sec}$. using a current of 120 milliamps. It is to be noted that if the burn is placed too far from the limbus in the rabbit, vascularization tends not to occur (Campbell and Michaelson, 1949).

This technique regularly produced an area of coagulation $3 \mathrm{~mm}$. in diameter through the whole thickness of the cornea, with a $1.5-\mathrm{mm}$. strip of clear cornea between the upper edge of the lesion and the nearest point on the limbus. In all cases, also, pigmentary changes occurred in the underlying iris, and an opacity in the underlying part of the lens. One eye only was used in each animal.

Three groups of fifteen rabbits each were treated in this way. The groups were subsequently divided as follows:

Group 1. Controls: no treatment.

Group 2. An injection of $2.5 \mathrm{mg}$. cortisone was given under the upper bulbar conjunctiva on the day of the burn and on alternate days up to the 20th day.

Group 3. An injection of $10.0 \mathrm{mg}$. cortisone was given under the dorsal skin, on alternate days for the first 4 days and subsequently daily up to the 20th day. (The 
omission of the injection on the first 2 alternate days was unintentional: this may have slightly affected the results, but not the deductions therefrom.)

\section{Results}

The average normal course of the control lesion, which was observed daily by a $\times 10$ loupe or slit lamp, showed that, after the development of an initial oedema and infiltration surrounding the lesion, superficial vessels began to grow from the neighbouring limbus at about $48 \mathrm{hrs}$. The vessels reached the lesion about the 5th or 6th day and thereafter spread into it. The advancing edge of the patch of vascularization showed small saccular aneurysms; loop formation was also observed. The character of the advancing edge of the vascularizing patch remained fairly constant until it had travelled about twothirds across the lesion, and the feeding vessels became progressively fewer but larger once the lesion had been reached. The whole reaction became less marked when the vessels were approximately two-thirds to three-quarters across the lesion; the progress of vascularization became slower, and thereafter the vessels gradually faded.

Figs 1, 2, and 3 illustrate the extent to which vascularization developed in each animal of each of the three groups. The circle in

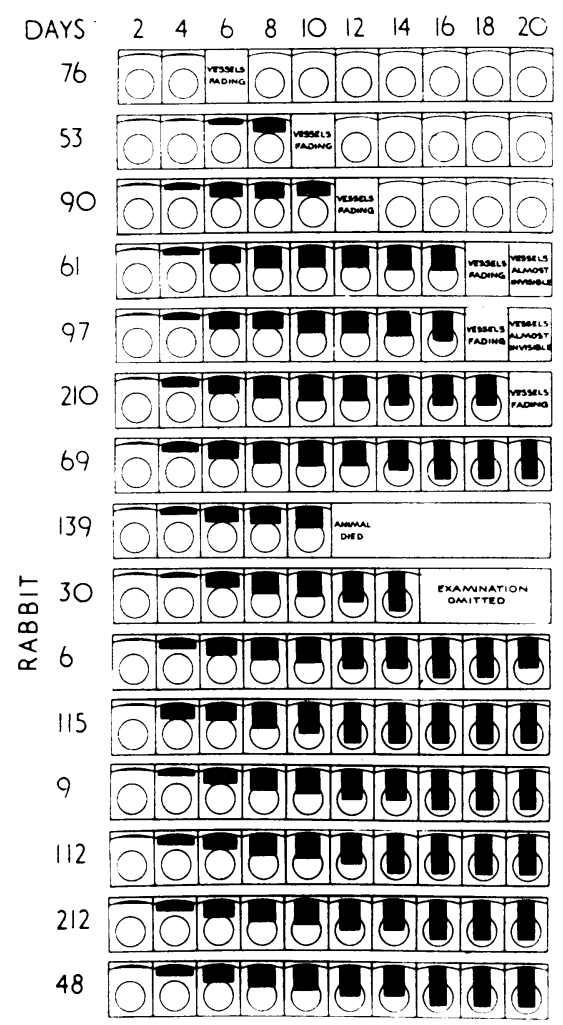

FIci. 1.- Chart showing extent and degree of vascularization in eyes of rabbits in control series.

each rectangle represents the lesion, and the arc above it the limbus. The extent of the shading indicates the number and size of the vessels; no shading implies, except where otherwise stated, that there were no vessels or that they were so short and attenuated as to be negligible. Figs 4 and 5 are typical photographs representative of the control group and of the group treated by subconjunctival injections, on the 6th and 14th day after burning respectively.

It is to be noted that extensive vascularization, which was 
remarkably constant in degree, occurred in all but two of the controls and was absent in ten of each of the cortisone groups: such a result is statistically significant*.

Apart from the extent of the vascularization, it was observed that in the majority of all cases a halo of infiltration surrounded the lesion

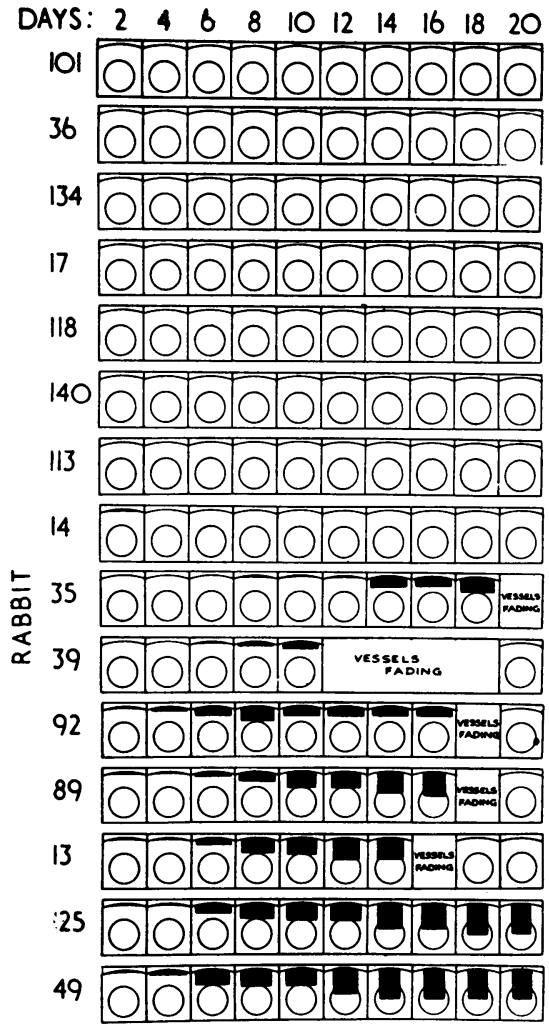

FIG. 2.-Chart showing extent and degree of vascularization in eyes of rabbits treated with subconjunctival cortisone.

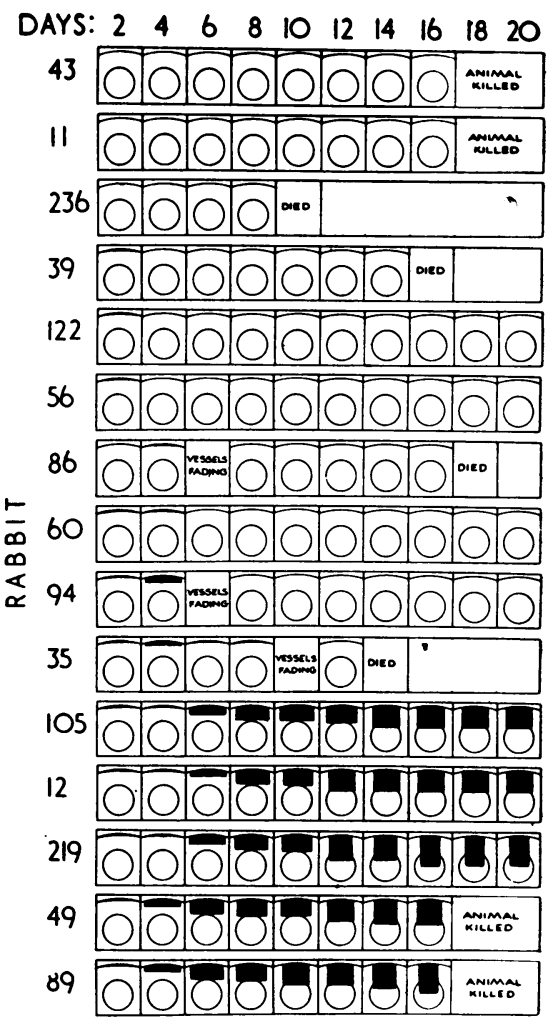

Fig. 3.-Chart showing extent and degree of vascularization in eyes of rabbits treated with systemic cortisone.

up to about the 10th day. This did not appear to be affected by cortisone; nor did the hormone appear to influence the rate at which the lesion became smooth and free from staining with fluorescein. In a number of cases a groove of varying depth developed on the upper half of the lesion, just inside and concentric with its upper edge, which impeded to some extent the advance of the vessels. In a few cases perforation took place, in which case the animal was replaced by another. Cortisone did not appear to influence the

* Such a sequence would occur by chance approximately only twice in a million sequences of fifteen. 

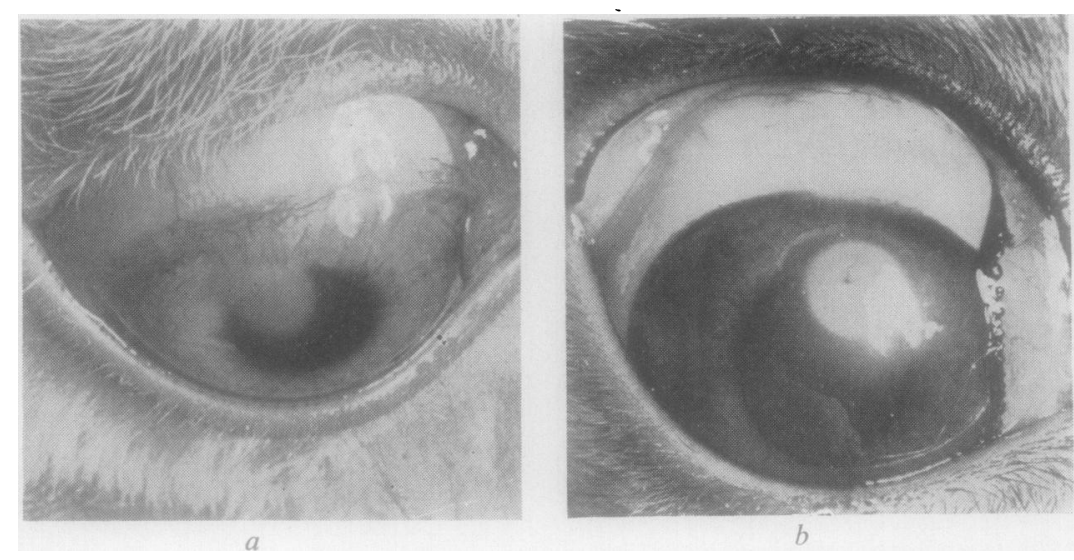

FIG. 4.-Photograph of representative corneal burns on 6th day. (a) Control. (b) After treatment by subconjunctival cortisone.
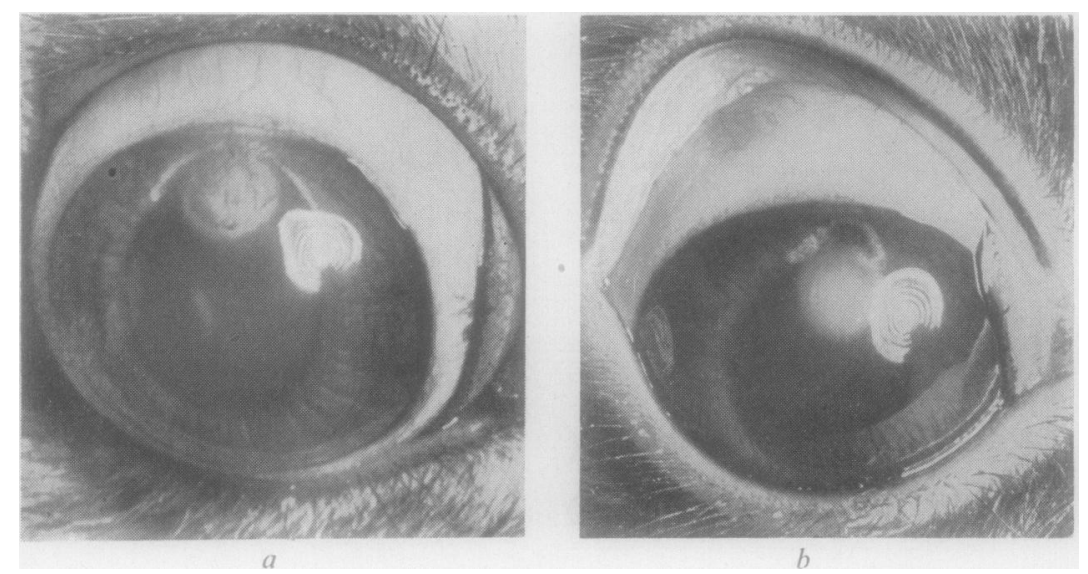

Fig. 5.-Photographs of representative corneal burns on 14th day. (a) Control. (b) After treatment with subconjunctival cortisone. (The "Placido" reflex is a photographic artifact.)

formation of this groove. In the group treated systemically, all the animals lost weight, and eight died or became so feeble that they had to be killed. Blood counts and metabolic tests were not carried out.

\section{Summary and Conclusions}

It is concluded that cortisone inhibits vascularization after experimental heat burns to the cornea of the rabbit. While there was no marked difference between the results in animals treated by sub- 
conjunctival injection and those in animals treated by parenteral injection, the former method has the advantage of having no systemic ill-effects. It would seem probable that the use of cortisone in clinical cases of this nature is justified.

The authors wish to express their thanks to the Medical Illustration Department of the Institute of Ophthalmology, London, for their help and co-operation in the preparation of the illustrations.

Part of the expense of this investigation was met from the Alexander Pigott Wernher Memorial Trust Fund.

The cortisone used in this investigation was supplied by the Medical Research Council and was in part derived from a gift from Merck and Co. made jointly to the Medical Research Council and the Nuffield Trust.

\section{REFERENCES}

Campbell, F. W., and Michaelson, I. C. (1949). British Journal of Ophthalmology, 33, 248

Jones, I. R., and Meyer, K. (1950). Proc. Soc. exp. Biol., N.Y., 74, 103. 